\title{
El teatro judío en la polémica shund/kunst: el IFT y el teatro de arte
}

\author{
Paula Ansaldo \\ Universidad de Buenos Aires \\ paulansaldo@hotmail.com \\ Artículo bajo licencia Creative Commons \\ Atribución 4.0 Internacional (CC BY 4.0) \\ ENVIADO: 2019-02-15 \\ ACEPTADO: 2019-05-31
}

\section{RESUMEN}

En este trabajo nos proponemos reflexionar sobre el movimiento de teatros de arte judíos que tuvo su auge a principios del siglo XX, con el objetivo de indagar en la forma en que sus postulados incidieron en la creación del teatro IFT en 1932 en Argentina. El surgimiento de Buenos Aires como un centro teatral judío de importancia fue tardío con respecto al desarrollo de las principales ciudades teatrales judías en Europa del Este, Rusia y los Estados Unidos, por lo que el teatro judío porteño heredó una serie de debates y polémicas que para entonces ya estaban firmemente instalados en el campo teatral judío, y que resultan fundamentales para entender el surgimiento de un teatro de arte en Buenos Aires.

\section{RESUMO}

Neste trabalho propomos refletir sobre o movimento dos teatros de arte judaicos que tiveram seu auge no início do século XX com o objetivo de investigar o modo como seus postulados influenciaram a criação do teatro IFT em 1932 na Argentina. O surgimento de Buenos Aires como um centro de importância teatral judaica tardou no que diz respeito ao desenvolvimento das principais cidades teatrais judaicas na Europa Oriental, Rússia e Estados Unidos, de modo que o teatro judaico de Buenos Aires herdou uma série de debates e controvérsias que a essa altura, eles estavam firmemente instalados no teatro judaico e são essenciais para entender o surgimento de um teatro de arte em Buenos Aires.
PALABRAS CLAVES

Teatro judío; Teatro IFT; Teatro de Arte; Buenos Aires.

\section{PALAVRAS-CHAVE}

Teatro judeu; Teatro IFT; Teatro de Arte; Buenos Aires.

\section{ABSTRACT}

In this essay we aim to explore the Jewish art theaters movement that had its peak in the first decades of the Twentieth century. We pretend to examine the way in which the Jewish art theaters postulates, influenced thecreation of the IFT Theater in Argentina when it was founded in 1932. The development of Buenos Aires as a Jewish theatrical center of international relevance was late compared to the main Jewish theatrical cities in East Europe, Russia and the United States, which experienced its moment of greatest splendor in the first decades of the Twentieth century. For that reason, the Jewish theater of Buenos Aires, inherited a number of debates and controversies that by then were already firmly installed in the Jewish theatrical scene and that are fundamental to understand the emergence of an art theater in Buenos Aires.

\section{KEYWORDS}

Jewish theater; IFT Theater; Art Theater; Buenos Aires. 


\section{INTRODUCCIÓN}

El gran auge del teatro judío comienza en nuestro país a partir de la década del 30 , pero el surgimiento de Buenos Aires como un centro teatral judío de importancia, es tardío con respecto al desarrollo de las principales ciudades teatrales judías en Europa del Este, Rusia y los Estados Unidos, que tienen su momento de mayor esplendor en las primeras décadas del siglo XX. Esto se debió a que durante el período de entre guerras y en la segunda posguerra, se asentó en Buenos Aires una gran población de judíos ídish parlantes ${ }^{1}$ que llegaron escapando del antisemitismo y las difíciles condiciones de vida en Europa, y que para ese entonces ya tenían restringido el ingreso a los Estados Unidos. Debido a esto, la ciudad se convirtió en un destino atractivo para intelectuales y artistas, ya que contaba con una rica vida cultural en ídish que estaba en pleno crecimiento. Esto provocó que el teatro judío porteño herede una serie de debates, posicionamientos y polémicas que para entonces ya estaban firmemente instalados en el campo teatral judío, y que se revitalizaron al desarrollarse en un nuevo contexto.

En este trabajo nos proponemos indagar en el movimiento de teatros de arte judíos que surge a principios del siglo XX, con el objetivo de reflexionar sobre cómo sus postulados incidieron en la creación, en 1932, de un teatro de arte judeo-argentino: el IFT (Idisher Folks Teater - Teatro Popular Judío). Se trata en este sentido, de un ejercicio de Teatro Comparado ${ }^{2}$, un abordaje que resulta necesario, dado que el teatro judío de Buenos Aires formaba parte durante este período de una red cultural transnacional, una red interconectada de comunidades de ídish parlantes alrededor del mundo. Los judíos que vivían en los diferentes países de la Diáspora, constituían en este sentido una audiencia global, no confinada por las barreras idiomáticas o geográficas que limitaban a los teatros nacionales, ya que el territorio del ídish era simplemente ídishland: todo lugar donde se hablaba ídish. Esto provocaba que los directores, actrices y actores judíos trabajaran de manera itinerante, desplazándose y viajando permanentemente entre los diferentes centros teatrales judíos del mundo: de Varsovia a París, de Vilna a Nueva York, de Londres a Buenos Aires.

Pero a pesar de ser una característica que particularizaba al teatro judío desde su surgimiento, la itinerancia fue percibida históricamente como uno de sus mayores puntos débiles, ya que se consideraba que su inestabilidad geográfica era uno de los principales motivos por los cuales el teatro judío no lograba desarrollarse en comparación con otros teatros nacionales. Esto se debía a que a causa de la ausencia de infraestructura nacional, ni los dramaturgos ni las compañías de teatro ídish tenían acceso a ningún tipo de subvención estatal, academia dramática, espacios teatrales establecidos $u$ otros beneficios con los que los teatros nacionales contaban. No obstante, nuevos investigaciones como las de Debra Caplan $(2013 ; 2014)$ re-conceptualizan lo que tradicionalmente había sido percibido como una flaqueza para convertirlo en una virtud, ya que sostienen que la itinerancia resultaba una ventaja artística, dado que era precisamente su carácter itinerante lo que les permitía alcanzar una audiencia global. Los artistas y las compañías de teatro judío funcionaban de esta forma como un conducto para que viajara de un lugar a otro repertorio dramático e ideas teatrales, en un período en que la comunicación y la lengua eran todavía un limitante para cualquier compañía.

Consideramos que debido a esto, el teatro judío funcionó como una corriente modernizadora que influyó notablemente en el campo teatral de Buenos Aires, ya que debido a su carácter itinerante y por vía del ídish, llegaron a la Argentina ideas teatrales, repertorios, poéticas de actuación y estéticas de vanguardia que aún no se habían desarrollado en los escenarios porteños

1 El ídish es la lengua hablada por la mayoría de la población judía de Europa Oriental hasta la Segunda Guerra Mundial

2 La disciplina que estudia "los fenómenos teatrales desde el punto de vista de su manifestación territorial (planeta, continente, país, área, región, ciudad, pueblo, barrio, etc.), por relación y contraste con otros fenómenos territoriales y/o por superación de la territorialidad” (Dubatti, 2012: 110) 


\section{EL MOVIMIENTO DE TEATROS DE ARTE JUDÍOS}

El teatro profesional aparece en el mundo judío solo después de que la secularización y los procesos de emancipación e integración comenzaran a transformar radicalmente la vida tradicional judía a partir del siglo XVIII. Desde mediados del siglo XIX, surgen prácticas teatrales seculares en toda Europa del este y Rusia; y en 1876 Abraham Goldfaden (1840 - 1908) funda la primera compañía teatral profesional judía.

Pero a diferencia de la literatura ídish, que desde finales del siglo XIX experimentaba un gran desarrollo -especialmente a través de la producción de Méndele Moijer Sforim (1835-1917), Sholem Aleijem (1859-1916) e Isaac Leibush Peretz (1851-1915)para principios del siglo XX, el teatro en ídish enfrentaba el grave problema de la ausencia de repertorio. En un momento en donde el teatro era valorado en función del texto dramático, la falta de repertorio y la ausencia de dramaturgos que desarrollaran una literatura dramática en ídish, era considerado una de las principales razones del atraso en el que se encontraba el teatro judío con respecto a lo que estaba sucediendo en los escenarios europeos contemporáneos. En este período, en lugar de crearse argumentos originales para la escena, la cartelera del teatro judío se abastecía mediante operetas y melodramas domésticos, que si bien satisfacían el gusto popular y tenían mucho éxito entre los espectadores, eran adaptaciones de historias preexistentes.

Para el grupo de escritores e intelectuales reunidos en Varsovia en torno a la figura del escritor I. L. Peretz esto era una verdadera preocupación, puesto que como señala Debra Caplan (2013: 40), consideraban que el teatro era el medio ideal para establecer la validez de la cultura judía moderna. Esto se debía a que incluso para los mejores escritores judíos era muy dificultoso llegar a los lectores no judíos puesto que escribían sus obras en el alfabeto hebreo. El teatro ídish, en cambio, al ser hablado y no escrito, poseía comparativamente una ventaja fundamental en este sentido. La escena ídish aparecía así como el espacio perfecto para transmitir la cultura judía moderna al público europeo, puesto que Peretz y sus colegas pensaban que si el teatro ídish lograba tener éxito con los espectadores no judíos, la legitimidad de la lengua, la literatura y la cultura ídish, estaría permanentemente asegurada. El teatro como lugar de encuentro físico, concreto, que implica necesariamente el convivio, es decir, la reunión de cuerpo presente entre espectadores y artistas, garantizaba así la llegada real a ese Otro que pretendían alcanzar.

Debido a estar razones, desde aproximadamente 1905 hasta el comienzo de la Primera Guerra Mundial, la pregunta por el teatro (la teater-frage) se convirtió en motivo de discusión y polémica permanente entre los intelectuales judíos polacos. Por primera vez, se comenzaron a escribir artículos y reseñas sobre teatro en los diarios judíos y progresivamente apareció una verdadera crítica teatral especializada. Alineados con las ideas renovadoras de Peretz, intelectuales y artistas -tales como el dramaturgo Jacobo Dinezon, el escritor A. Vayter y el crítico teatral Alexander Mukdoyni- se dedicaron a escribir acerca de la necesidad de reformar el teatro de forma completa para crear un nuevo teatro ídish que estuviese dedicado a altos ideales artísticos.

Estos autores se basaban en la distinción extrema entre formas teatrales consideradas buenas o malas, altas o bajas, polarización que fue formulada a partir de los términos contrapuestos de Kunst (Arte) y Shund, una palabra que utiliza por primera vez en 1893 el editor y periodista Abraham Cahan para referirse a lo que él deseaba que fuera un género en desaparición: las novelas populares ídish. La palabra -reapropiada por el campo teatral- connotaba la idea de un arte basura, bajo, vulgar; en oposición al teatro de arte, elevado, refinado, intelectual y literario, que se aspiraba a alcanzar.

Shund era básicamente el concepto que los críticos utilizaban para caracterizar prácticamente a todo el teatro judío pre-existente, al cual consideraban vulgar y efectista, en tanto tenía como objetivo satisfacer el gusto popular, "agradar a Moishe" como solía decir la prensa teatral del período refiriéndose a los espectadores de mente simple, ingenuos y poco cultivados. Las críticas se focalizaban también en la actuación, que evaluaban de muy baja calidad en tanto la mayoría de los actores judíos tenían muy poca educación y prácticamente ningún entrenamiento formal. Por otro lado, el tiempo de los ensayos era muy breve, por lo que debían recurrir a la utilización de recursos propios del actor popular, como la improvisación y los shtiks (rutinas cómicas asociadas a un actor o actriz en particular), para compensar posibles faltas y errores durante la representación.

En cuanto a las temáticas, hemos señalado que los melodramas domésticos eran los que dominaban el repertorio de la mayoría de las compañías, así como las llamadas "comedias de lágrimas" (o coloquialmente "obras cebolla") que intercalaban elementos alegres y tristes en igual proporción, puesto que el objetivo era divertir y emocionar a la vez. Como hemos dicho, todas las obras incluían momentos de baile, música y canto, siendo esto parte fundamental del espectáculo y del éxito entre el público, para quienes la calidad literaria o el realismo de la representación eran aspectos de importancia secundaria, ya que la atención estaba puesta en los elementos espectaculares como la actuación y el canto.

Por esta razón, los críticos culpaban al shund de corromper el gusto del público, y vinculaban metafóricamente el teatro judío con la imagen de un niño al que había que ayudar a madurar y crecer. Como ha señalado Nina Warnke (2008), en la retórica típica de la época frecuentemente aparecía la idea de una evolución lineal de las culturas hacia un estado cada vez más desarrollado en el que la cultura judía se encontraba largamente retrasada, razón por la cual, los intelectuales establecieron una relación paternal con este niño que necesitaba constante vigilancia y guía para crecer como una institución respetable y madura. Y esto se hizo extensivo también a las audiencias que debían aprender a valorar otro tipo de espectáculos de mayor calidad artística. En este sentido, Peretz y su círculo creían que el teatro tenía la función de educar a los espectadores, como lo indica su famosa frase (grabada muy posteriormente en una de las paredes del IFT) "Teater: a shul far dervaksene": el teatro es escuela para adultos.

Es así que, a partir del siglo XX, incentivados por el interés despertado en los círculos intelectuales y por el surgimiento de una audiencia más cultivada, y gracias a la creciente estabilidad profesional del teatro ídish, surge una nueva generación de autores teatrales que escriben obras originales que abordan temáticas específicamente judías. A los escritores I.L. Peretz y Sholem Aleijem, que ya habían incursionado en el género dramático, se le sumaron en este período los autores: León Kobrin (1873-1946), Sholem Asch (1880-1957), David Pinski (1872-1959), H. Leivick (1888-1962), Osip Dimov (1878-1959) y Peretz Hirschbein (1880-1948), entre los más prolíficos, quienes desarrollaron una producción teatral en ídish que dio lugar finalmente a la constitución de un repertorio teatral judío, una 
dramaturgia que buscaba reflejar y lidiar con la experiencia judía por medio de un lenguaje propio y original.

Pero dentro del circuito teatral judío, las obras de estos autores muchas veces no llegaban a ser representadas en el teatro comercial, debido a que la audiencia seguía demandando elementos espectaculares, como la música y el baile, del que este teatro literario carecía. Por lo que muchas de estas obras eran únicamente publicadas como literatura dramática sin llegar nunca a los escenarios.

Es por este motivo que comienzan a surgir compañías teatrales independientes que buscaban poner en escena un repertorio compuesto únicamente por obras de alto nivel literario, y que se identifican a sí mismas con el concepto de Kunst Teater, siguiendo en este sentido, el modelo del Teatro de Arte de Moscú dirigido por Constantin Stanislavsky.

La primera compañía independiente surge en 1908 en la ciudad de Odessa, Rusia: se trata de la Hirschbein Troupe dirigida por el dramaturgo Peretz Hirschbein. Estaba conformada por actores muy jóvenes y carecía de una estructura verticalista organizándose en cambio a la manera de un ensamble, con roles intercambiables y sin un director artístico definido. Pero su principal característica -que los diferenciaba de otras compañíasera su repertorio de teatro exclusivamente literario, compuesto por obras de autores como Sholem Aleijem y Sholem Asch, y piezas escritas por el mismo Hirschbein. La compañía, que encarnaba las ideas renovadoras de Peretz, posibilitó la difusión de estos nuevos autores dado que durante dos años realizaron giras por Europa del Este, pero su debut en Varsovia no tuvo el éxito esperado y la compañía se disolvió en julio de 1910. Sin embargo, a pesar de su "fracaso", la Hirschbein Troupe contribuyó a crear un nuevo entusiasmo en la audiencia con respecto al teatro ídish y estableció los fundamentos sobre los que luego se basaría el movimiento de teatros de arte que surge en el período de entreguerras.

En este sentido, la Hirschbein Troupe se considera un antecedente fundamental de una de las compañías de mayor renombre e importancia en la historia del teatro de arte judío: la Vilner Troupe. Ésta se funda en 1915 en la ciudad de Vilna, y como muchos de estos primeros teatros hunde sus raíces en los grupos de teatro amateur (en ídish "libhober bines", literalmente "aquellos que aman el escenario"), que desde finales del siglo XIX habían surgido a lo largo de Europa del Este. Se trataba de organizaciones que reunían en su mayoría a actores jóvenes, que estaban interesados en poner en escena dramas de alto valor literario. Estos clubes funcionaron como un espacio de entrenamiento y educación para aquellos que tenían aspiraciones artísticas, al darles la posibilidad de desarrollar una experiencia en el escenario, y de alguna manera compensaron así la falta de una escuela de teatro oficial que pudiese formar a las nuevas generaciones de actores y actrices. Por otro lado, permitieron que se representaran obras de dramaturgos incipientes que no tenían acceso al circuito comercial, y acercaron el público a estilos teatrales nuevos y textos dramáticos diferentes a los que estaban acostumbrados a ver en los escenarios profesionales.

Estas experiencias sentaron de esta manera, las bases sobre las que se sustentaría la Vilner Troupe, que nace a partir del grupo amateur denominado FADA (Farein fun idishe dramatishe artistn - Agrupación de Artistas Dramáticos Judíos). Seguimos a Debra Caplan, que ha estudiado en profundidad la historia de la compañía (2013; 2014), y considera que la Vilner Troupe fue el epicentro del movimiento internacional del teatro de arte judío, y que fue gracias a su gran proyección transnacional que las poéticas de vanguardia lograron finalmente una aceptación al interior del campo teatral judío. Este cambio comienza a darse a partir de 1920 con el estreno de la obra El Dibuk de Shlomo Anski, que marca un punto de inflexión en la estética desarrollada por la compañía. A partir de 1924 comienza lo que Caplan denomina el paradigma transnacional de la compañía, ya que durante estos años los miembros actuales y pasados de la Vilner Troupe podían ser encontrados actuando en cinco continentes. Aparece entonces la denominación "vilner", es decir, "miembro de la Vilner Troupe", nombre que les brindaba a sus integrantes una reputación, una suerte de identidad artística que los identificaba con una manera de hacer teatro que aparecía como nueva, moderna y de alta calidad.

De esta manera, la itinerancia les permitió ampliar su audiencia y su llegada a distintos espectadores, inspirando a otros actores y actrices judías a crear sus propios teatros de arte. Es así que, inmediatamente después de la Primera Guerra Mundial, comenzaron a aparecer compañías teatrales judías que se autoproclamaban de teatro de arte. En Varsovia, Zygmunt Turkow creó el Varshever Yidisher Kunst Teater - VYKT (Teatro Judío de Arte de Varsovia). En Nueva York, Maurice Schwartz fundó en 1918 su Yiddish Art Theater y en 1919 Jacobo Ben-Ami (ex miembro de la Hirschbein Troupe) creó, también en Nueva York, el Jewish Art Theater, afirmando así, una manera de hacer teatro que se difundió a lo largo de todo el campo teatral judío durante el período de entre guerras. 


\section{BUENOS AIRES COMO UN CENTRO TEATRAL JUDÍO}

Tanto Nahshon (2004) como Sandrow (1995) han señalado la paradoja de que el momento en el que el teatro ídish norteamericano alcanza su más alto nivel estético, coincide con la pérdida de la llegada a las masas. La audiencia del teatro ídish en Estados Unidos decae drásticamente a partir de la década del 30, debido a que por un lado, se limita la inmigración judía que podía brindarle nuevo público a los espectáculos; y por el otro, las generaciones de jóvenes nacidos en el país estaban ya asimilados, de manera que el ídish fue perdiendo su lugar como primera lengua entre los judíos norteamericanos. Por esta razón, la temporada teatral se volvió cada vez más corta, muchos teatros cerraron, y los actores optaron por irse de tour a otros lugares del mundo en donde las comunidades judías estaban en pleno crecimiento, como era el caso de la Argentina, convirtiéndose así en actores itinerantes.

Esto provoca un cambio en la cartografía teatral judía, que posiciona a Buenos Aires como un centro teatral de importancia, debido no solo a su población de judíos ídish parlantes que podía brindarle a los teatros una audiencia importante y permanente, sino a que el hemisferio Sur se beneficiaba por la oposición de temporadas, de manera tal que en el receso de verano, los actores extranjeros podían venir a trabajar a la Argentina. Es debido a esto, que si bien el teatro ídish surge en Argentina a principios de Siglo XX, ubicamos su momento de mayor esplendor a partir de la década del 30, cuando Nueva York pierde su lugar central en la cartografía teatral judía, en beneficio de nuevas territorialidades.

En este período, cuatro teatros de Buenos Aires estaban completamente destinados a presentar obras en ídish: el Excelsior y el Soleil en el barrio de Abasto, el Mitre en Villa Crespo y el Ombú en el barrio de Once. El teatro empresarial en ídish de Buenos Aires, se organizaba en función de un Star System, un sistema de estrellas que se sostenía gracias al actor central que venía del extranjero, completando el elenco con actores locales. Los empresarios traían de esta forma figuras extranjeras, tales como Jacobo Ben Ami, Maurice Schwartz y Joseph Buloff, que garantizaban un éxito seguro de la temporada gracias a su fama internacional.

Pero a medida que el teatro judío de Buenos Aires se fue desarrollando, esta forma de funcionamiento comenzó a ser cada vez más cuestionada, especialmente debido a la fuerte dependencia que sufría el campo teatral judío local con respecto a las visitas de las figuras extranjeras. Los actores y actrices argentinos, no querían verse obligados a esperar a la llegada de las estrellas para poder actuar en obras de repertorio, especialmente cuando la alternativa era participar en pequeñas piezas musicales, operetas o espectáculos de vaudeville que incluían canciones, baile y algunas veces un humor chabacano. Debido a esto, muchos críticos de la época, caracterizaban a estos espectáculos como shund teater, recuperando algunos años después, la terminología de la mencionada campaña de Peretz por un teatro de arte judío.

El empresario teatral Adolfo Mide recuerda en sus memorias (1957) que la discusión sobre los defectos y beneficios de este sistema, los llevó incluso a realizar un "juicio al Star system" en el Teatro Ombú en 1932. En este juicio intervinieron Pinie Katz, redactor del diario Di Presse como juez a cargo del tribunal, y el crítico teatral del Di Presse el Dr. L. Zitnitzky como fiscal. Unos años después, ambos jugarían un importante rol en el IFT, siendo Zitnitzky su primer presidente. Los dos eran intelectuales de izquierda que escribían artículos en los diarios judíos abogando por la elevación artística del teatro judío argentino, y que -tal como habían hecho sus colegas norteamericanos y polacosenarbolaban el concepto de kunst teater para contrarrestar la tendencia hacia el shund que primaba en los escenarios porteños. 


\section{EL IFT, UN KUNST TEATER}

Es en el contexto de este debate, que en 1932 se funda el IDRAMST (Idishe Dramatishe Stude - Estudio Dramático Judío). En este primer período, el Estudio se creó como una escuela teatral para formar artistas que quisieran hacer un mejor teatro. Pero debido a la gran convocatoria que tuvieron sus representaciones, sus integrantes adquirieron temprana consciencia de la importancia de crear una compañía teatral judía que estuviese compuesta por actores y actrices del medio local, y que pudiese funcionar de manera estable. Por lo que en 1936 iniciaron una campaña por un Teatro Popular Judío en Argentina, que dio lugar en 1937 a la transformación del IDRAMST en IFT.

Los miembros de este primer grupo consideraban que existía un vacío a llenar en el campo teatral judío de Buenos Aires, debido a la ausencia de un kunst teater, de una compañía teatral que tuviese un fuerte compromiso estético, y cuya principal preocupación fuese la de poner en escena obras de alto nivel artístico y literario. Al igual que el Teatro del Pueblo, fundado por Leónidas Barletta en 1930, creían firmemente en que para que esto fuese posible, este teatro tenía que ser independiente en primer lugar de la taquilla, ya que el deseo de obtener ganancias, limitaba la posibilidad de experimentar y de tomar riesgos artísticos. En un artículo titulado justamente "Del shund al kunst", publicado en marzo de 1936 en el n० 4 de la revista Nai Teater (Nuevo Teatro) -el órgano de difusión del IFT- G. Dorín sostiene:

El teatro profesional, que en los países capitalistas es un negocio, no para el actor sino para el empresario, tiene en cuenta los intereses de la ganancia en su objetivo final. $Y$ donde la Caja dicta, no hay lugar para el arte ni para los ideales. La industria teatral burguesa, que trabaja para el ancho e ignorante mercado, busca a la masa, no para elevarla y educarla, sino para satisfacerla livianamente y que vuelva pidiendo más³.

Como puede verse, los integrantes del IFT concebían el teatro como una herramienta para movilizar las conciencias de los espectadores, tal como lo proponía Romain Rolland, y entendían que solo a través del desinterés económico, podrían cumplir su objetivo de crear un teatro que satisficiera las necesidades del pueblo.

Pero si bien podemos considerar al IFT como un teatro popular -tal como lo indica su nombre- en cuanto a su propósito de educar a las masas, es necesario pensarlo también como un teatro de arte, debido a su búsqueda de modernización artística en cuanto a su repertorio, su poética de puesta en escena y el estilo de actuación de su elenco. Esta identificación con ideales no solo políticos sino también artísticos, era explícitamente enunciada por ellos mismos en sus campañas para recaudar dinero o conseguir más socios para la institución, donde se auto denominaban como un "teatro moderno y experimental". Al caracterizarse de esta manera, pretendían recibir el apoyo de todo aquel que se considerara defensor del kunst teater, dirigiéndose en sus comunicados alternativamente a los "amigos del teatro de arte" o a los "amantes de un mejor teatro". Asimismo, finalizaban gran parte de sus publicaciones llamando a la población judía a asociarse y de esta manera cumplir "con 3 Todas las traducciones del ídish al castellano de los artículos de la IFT que nunca llegó a finalizarse, traduciendo gran parte de los números de la revista Nai Teater al español. La misma puede consultarse en la Fundación IWO de Buenos Aires un gran deber para con el arte y la cultura judía”. Cimentaban así la idea, de que apoyar al IFT era colaborar con el único teatro que podía elevar el nivel de la cultura judía en Buenos Aires, y sustentar así la única compañía que en ese momento encarnaba las ideas renovadoras que habían llevado al movimiento de teatros de arte judíos a florecer a lo largo del mundo.

De esta forma, los intelectuales y críticos que rodeaban al IFT, erigieron la campaña para fundar un teatro estable a partir de la ya probada retórica de principios del Siglo XX, que se basaba en la dicotomía shund/kunst. Buscaron así instalar en el imaginario la idea de que prácticamente todo el teatro comercial judío tenía que ser considerado shund, y que luchar contra él era una tarea que tenía que llevar adelante toda la comunidad judía progresista de la Argentina, mediante su apoyo al Teatro IFT. En el mismo artículo ya citado, G. Dorín por ejemplo sostiene:

Un siglo atrás se logró echar al shund de la literatura judía, sin embargo, como revancha, el shund echó a la literatura del teatro. En el teatro nacional, el shund da el tono, ahí es el patrón. Es cierto que en el teatro, Mr. Shund adorna su cabeza vacía con moderno sombrero de copa, se pone una vestimenta de payaso con artísticos lunares, tapa sus pavadas con un chiste barato, con un cantito, con un bailecito, con una piernita desnuda. Se llama opereta, melodrama, vodevil, farsa, revista. Aparece bajo un nombre literario, pero es pura sangre de shund, porque está vacío de cualquier contenido, porque se adapta al más bajo gusto, porque solo tiene la intención de hacer cosquillas y de acariciar los más primitivos sentimientos y los más bajos instintos.

Pero la independencia de la taquilla no era suficiente para alejarse del shund. Los miembros del IFT, buscaban también independizarse de las estrellas, de las figuras extranjeras y del personalismo que primaba en el teatro empresarial judío. Siguiendo el modelo de las compañías teatrales judías de Europa, confiaban en la organización colectiva como pilar del nuevo teatro que querían crear, rompiendo así con la lógica del starism, un término proveniente del ídish que designaba el rol central que ocupaban los actores en el teatro comercial judío. El peso otorgado a la star, es denunciado en la revista Nai Teater $n^{\circ} 8$ de septiembre de 1937 por M. Lev, secretario del IFT:

Se sabe que todo actor debe cultivarse, de lo contrario es tragado por sus propias ambiciones. Cosa que no debiera ocurrir con los grupos de aficionados que se desarrollan en todo el mundo. Porque el único medio que tiene la facultad de desarrollarse, y desarrollar a su vez a los talentos, es el teatro social o grupal. Un ejemplo de este caso es Maurice Schwartz. Nadie dudaría de su talento y temperamento. Sin embargo, aparte de su personal influencia, su teatro en Nueva York es una ficción, no es un teatro con rostro propio. Si él se va, adiós teatro de arte, y cuando vuelve reúne un elenco y de nuevo es un teatro artístico.

La voluntad del IFT de constituirse como un teatro colectivo, respondía por un lado a la ideología de izquierda de los integrantes de la institución, que se basaba en la horizontalidad, el trabajo en grupo y la discusión en asamblea. Por el otro, seguía los lineamientos estéticos de los teatros de arte europeos como la Vilner Trupe y sobre todo, del teatro soviético GOSET (acrónimo ruso con el que era conocido el Teatro Estatal Judío de Moscú), que ponían el acento en el trabajo del ensamble por sobre las figuras individuales. Debido a esto la construcción grupal se convirtió en un elemento que caracterizó la composición es- 
tética de las representaciones del IFT, ya desde sus primeros tiempos.

Es así que para los integrantes del IFT, la creación de un kunst teater, no se limitó a elevar y enriquecer el nivel literario del teatro judío en Argentina, sino que se propuso también modernizar las formas, a partir de las nuevas tendencias que se estaban desarrollando en Europa. Como hemos dicho, estas corrientes innovadoras llegaron al IFT debido al flujo transnacional de directores, actrices y actores judíos itinerantes, que trajeron bajo el brazo ideas teatrales de vanguardia con las que habían tomado contacto en las compañías teatrales europeas de las que provenían.

En este sentido, no podemos dejar de señalar la importancia fundamental de David Licht, quien fue el director artístico de la compañía durante más de quince años, desde 1938 hasta 1953. Licht había formado parte de la Vilner Trupe y había dirigido en París el PIAT (Pariser Idisher Arbeter Teater - Teatro Obrero Judío de París), un teatro popular judío como el que el IFT estaba intentando crear. Bajo su guía, la compañía desarrolló una poética de puesta en escena que los singularizó profundamente dentro del campo teatral, no solo judío, sino también dentro de la escena argentina en general.

Siguiendo la herencia que traía de la Vilner Trupe, Licht desarrolló una estética cercana a la de un "modernismo fusionado" (Caplan, 2013), ya que se trataba de un modernismo inclusivo, que no tenía antagonista ni se oponía a la tradición sino que la integraba, de modo tal que podía tomar inspiración de la historia y el folklore judío pero sin resignar por ello la utilización de procedimientos provenientes de las poéticas de modernización europeas. Es así que, durante estos años, el elenco del IFT montó obras de temática judía, muchas de las cuales eran adaptaciones de cuentos y novelas de los más célebres escritores judíos, tales como Sholem Aleijem, I.L. Peretz, H. Leivik, entre otros autores que habían escrito muy poco teatro. En este sentido, la tarea de Licht no fue únicamente elegir un repertorio, sino crearlo. De esta modo, en lugar de luchar contra la tradición o negarla, sus adaptaciones incorporaban personajes clásicos del folklore y la literatura judía, poniendo el acento en lo que en ellos había de universal. Rosa Rapaport, integrante del elenco, señala al respecto que con Licht las tradiciones y el folklore judío aparecieron por primera vez en escena expuestos artísticamente ${ }^{4}$.

A su vez Licht montó obras de reconocimiento internacional en traducciones al ídish, tales como Los bajos fondos de Máximo Gorki en 1942, Todos los hijos de Dios tienen alas de Eugene O’ Neill en 1945 y Todos eran mis hijos de Arthur Miller en 1950, dando a conocer por primera vez estas obras al público judío. La difusión de obras universales era una función que el IFT consideraba primordial en tanto educador de las masas, ya que dado que la gran mayoría de los judíos hablaban en ídish, era fundamental ponerlos en contacto con la dramaturgia universal, traduciéndola a su idioma.

Por otro lado, Licht aportó innovaciones en cuanto a la poética de actuación del elenco y una nueva pedagogía en la formación de actores y actrices, que para esa época aún no estaba difundida en la Argentina: el sistema de Stanislavsky. Uno de sus primeros artículos sobre teoría teatral publicados en Nai Teater, es de hecho un recorrido desde el naturalismo hasta las teorías Stanislavskianas y la búsqueda de un nuevo realismo. La actriz argentina Cipe Lincovsky, formada bajo su dirección, recuerda en este sentido:

Él venía del Vilner trupe, con toda la escuela de Stanislavsky. Claro, él nos dirigía y nos llevaba a situaciones, nos pedía situaciones, y muchos años después, cuando él ya había muerto y salió el primer libro del método Stanislavsky, nosotros nos dimos cuenta de que todos habíamos actuado bajo ese método, y por eso el IFT era tan especial (...) Era otro tipo de actuación moderna, totalmente. Pero era porque todo esto que leíamos en el libro: memoria emotiva, memoria emocional, sensitiva, todo eso lo habíamos hecho nosotros (McGee Deutsch, 2010: s/n).

Es importante destacar, que en la Argentina los escritos de Stanislavsky no se publicaron traducidos al español hasta la década del 50, y su pensamiento no se difundió ampliamente en la escena porteña hasta la llegada de Hedy Crilla, que fundó en 1947 una escuela teatral en la Sociedad Hebraica Argentina, y que en 1958 comenzó a trabajar junto al grupo teatral independiente La Máscara, popularizando allí la pedagogía Stanis. lavskyana.

En el IFT sin embargo, ya desde la llegada de David Licht en 1938, y especialmente con la fundación de la Escuela Dramática en 1947 , se comenzaron a formar actores y actrices en la búsqueda de un realismo que no estuviese centrado en la forma exterior de la escena, sino en la búsqueda de la verdad interior, es decir, un realismo donde lo importante no era que fuesen verdaderos los decorados o la utilería, sino el sentimiento del actor. Estas nuevas formas de entender el teatro y la actuación, no fueron solamente transmitidas a las nuevas generaciones que se formaron en la Escuela del IFT, sino que se debatieron y analizaron tanto al interior del elenco, como de forma abierta al público general mediante conferencias, charlas, debates y publicaciones especializadas sobre historia y teoría teatral en la revista Nai Teater.

Esta forma innovadora de actuación del elenco, sumado a las puestas en escena modernas y a un repertorio original, provocó que durante estos años asistieran a las funciones del IFT directores, actrices y actores de la escena nacional, a pesar de que no comprendían el ídish. En numerosos documentos de la época, se señala que el elenco tenía que realizar funciones especiales a la medianoche, para permitir que los actores del teatro nacional, pudieran asistir después del horario en el que trabajaban en sus respectivos elencos. Esto sucedía ya desde 1938 cuando tuvieron que programarse funciones extraordinarias de la obra Boitre, el salteador judío de Moyshe Kulbak, la pieza con la que David Licht realizó su debut en Argentina. Y esta práctica siguió siendo habitual a lo largo de los años, especialmente en las ocasiones en que el IFT montaba traducciones al ídish de obras de dramaturgia moderna que aún no habían sido representadas en castellano. El caso más emblemático en este sentido, es tal vez el de Madre Coraje de Bertolt Brecht en 1953, ya que se trató del primer estreno de una obra de Brecht en Argentina, un año antes de la famosa puesta que realizó Nuevo Teatro de esa misma pieza.

De esta forma, el IFT cumplió no solo su objetivo inicial que pretendía elevar el nivel del teatro judío en Buenos Aires y alejar así a los espectadores del shund teater, sino que se transformó en un teatro de arte argentino, que con sus puestas en escena, su Escuela Teatral, sus conferencias y sus publicaciones, introdujo en la escena nacional poéticas teatrales innovadoras que tan sólo algunos años después, se volverían centrales en el campo teatral de Buenos Aires. 
Ansaldo, P. (2018). Teatro popular, teatro judío, teatro independiente: una aproximación al Idisher Folks Teater (IFT). Culturales, (6), e.345, México.

Berkowitz, J.; Henry B. (eds.). (2012). Inventing the Modern Yiddish Stage: Essays in Drama, Performance, and Show Business. Detroit: Wayne State University Press.

Caplan, D. (2013). Staging Jewish Modernism: The Vilna Troupe and the Rise of a Transnational Yiddish Art Theater Movement. Tesis doctoral, Harvard University.

Caplan, D. (2014). Nomadic Chutzpah: The Vilna Troupe's Transnational Yiddish Theatre Paradigm, 1915-1935. Theatre Survey, (55), 296-317.

Dubatti, J. (2012). Introducción a los estudios teatrales. Propedéutica. Buenos Aires: Atuel.

Dubatti, J. (2013). Cien años de teatro argentino: del Centenario a nuestros días. Buenos Aires: Biblos.

Fishman, D. (2005). The rise of modern Yiddish culture. Pittsburgh: University of Pittsburgh Press.

Fukelman, M. (2017). El concepto de "teatro independiente" en Buenos Aires, del Teatro del Pueblo al presente teatral: estudio del período 1930-1944, Tesis doctoral inédita.

Gorin, B. (1918). Di geshikhte fun idishn teater [La historia del teatro ídish], 2 vols., Nueva York: Literarisher Farlag.

McGee Deutsch, S. (2010). Entrevista a Cipe Lincovsky. Archivo Oral Centro Mark Turkow.

Mide, A. (1957). Episodios del teatro judío. Traducción de L. Skura y S. Skura. Buenos Aires.

Nahshon, E. (2004). The Yiddish Theater in America: a brief historical overview. En Baker, Z. M.; Sohn, B. (eds.), The Lawrence Marwick Collection of Copyrighted Yiddish Plays at the Library of Congress: An Annotated Bibliography, Washington: Library of Congress.

Risetti, R. (2004). Memorias del teatro independiente argentino 1930-1970. Buenos Aires: Corregidor.

Sandrow, N. (1995). Vagabond Stars: A World History of Yiddish Theater. Syracuse: Syracuse University Press.

Visacovsky, N. (2016). Argentinos, judíos y camaradas: tras la utopía socialista. Buenos Aires: Biblos.

Warnke, N. (2008). The child who wouldn't grow up: Yiddish Theatre and its critics. En: Berkowitz, J. (ed). Yiddish Theatre: New Approaches, Oxford: Littman Library of Jewish Civilization.

\section{OTRAS FUENTES}

- Comunicado de la Campaña por un Teatro Popular en Argentina, Buenos Aires, 1936, Archivo Pinie Katz.

- Carta a los amigos del teatro de arte, Buenos Aires, 1936, Archivo Pinie Katz.

- Carta a los amantes de un mejor teatro judío, Buenos Aires, 1937, Archivo Pinie Katz.

- Revistas Nai Teater (Nuevo Teatro), Archivo Fundación IWO. 
